\title{
Hydrodynamic coupling between two fluid membranes
}

\author{
Sanoop Ramachandran and Shigeyuki Komura \\ Department of Chemistry, Graduate School of Science and Engineering, \\ Tokyo Metropolitan University, Tokyo 192-0397, Japan \\ E-mail: komura@tmu.ac.jp
}

\begin{abstract}
The coupled in-plane diffusion dynamics between point-particles embedded in stacked fluid membranes are investigated. We calculate the contributions to the coupling longitudinal and transverse diffusion coefficients due to particle motion within the different as well as the same membranes. The stacked geometry leads to a hydrodynamic coupling between the two membranes.
\end{abstract}

Biological membranes are fundamental to the existence of life with their ability to separate the "in" and "out" of a cell. Typical membranes are composed of lipid molecules which can spontaneously self-assemble into a fluid bilayer structure [1]. Under normal physiological conditions, finite temperature induces Brownian motion of membrane constituents, resulting in diffusive transport. In the seminal paper by Saffman and Delbrück [2], the diffusion coefficient of a rigid protein in a membrane was calculated. Following this work, there have been theoretical studies on diffusion of a rigid disk on supported membranes [3], a liquid domain in monolayers [4, 5], and a rod on immersed membranes [6, 7] or on Langmuir monolayers [8].

The present work focuses on the coupling of diffusion dynamics between two fluid membranes through an intervening bulk fluid. It was reported that model experimental systems in which two lipid membranes are stacked on a substrate exhibit correlated dynamics [9]. This planar geometry has become favorable to study the membrane dynamics which are otherwise not possible in vesicles [10. The coupling effect between two membranes can be important in biological systems with large concentration of cells such as in tissues. Other examples are Gram-negative bacteria which enclose a periplasmic space with an approximate width of about 15 to $20 \mathrm{~nm}$ between their inner and outer lipid bilayers [1]. Highly folded membranous organelles such as Golgi apparatus also correspond to a situation in which membranes come in close proximity to each other. In all these cases, it is very relevant to consider the hydrodynamic coupling between two biomembranes.

Hydrodynamic models of membranes exploit their fluid nature, in which the membranes are assumed to be two-dimensional (2D) viscous fluid sheets embedded in a three-dimensional (3D) solvent. One such investigation involved the calculation of the correlated diffusion of proteins embedded in a membrane immersed in an unbounded 


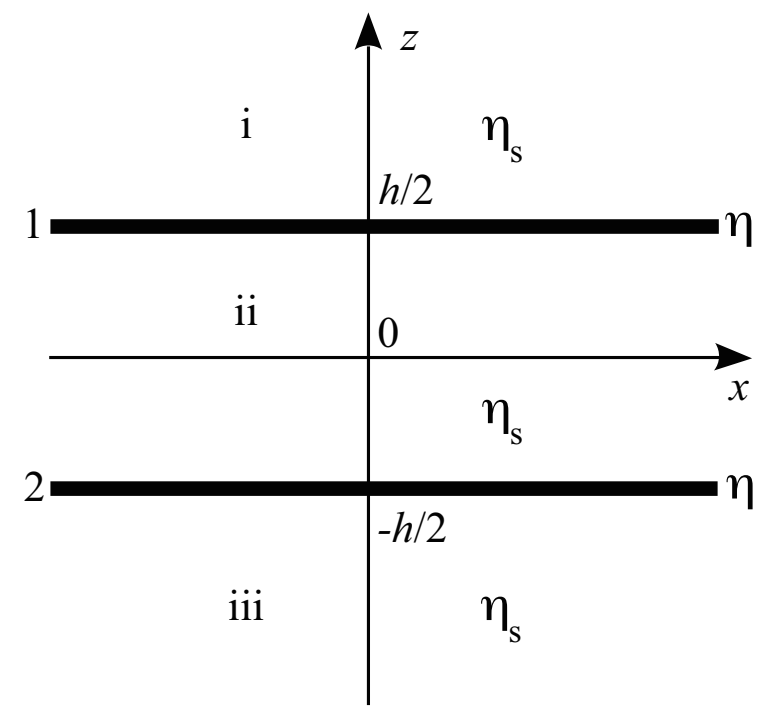

Figure 1. Schematic picture showing a set of stacked fluid membranes (solid thick lines) having $2 \mathrm{D}$ viscosities $\eta$ located at $z= \pm h / 2$. The two membranes are denoted by the labels 1 and 2 . The solvent of $3 \mathrm{D}$ viscosities $\eta_{\mathrm{s}}$ are labeled by the regions i, ii and iii.

fluid [11] or a membrane adjacent to a solid support [12]. In this Communication, we report on the coupling diffusion coefficients of particles embedded in such a stacked membrane system.

We first establish the governing equations. It is assumed here that the membranes are infinite planar sheets of fluid. The persistence length is defined as the length over which the correlations of the normal vector decay to zero [13]. For a lipid bilayer membrane with bending rigidity of about $10 k_{\mathrm{B}} T$, the persistence length turns out to be much larger than the size of a typical cell. Hence we neglect out-of-plane fluctuations of the membrane for mathematical simplicity. The fluid membranes are embedded in a bulk solvent such as water or suitable buffer solution. As shown in figure 1, the membranes are fixed in the $x y$-plane at $z= \pm h / 2$. Let $\mathbf{v}^{(i)}(\mathbf{r})$ be the $2 \mathrm{D}$ velocity of the membrane fluids. Here the index $i=1,2$ represents the two membranes, and the $2 \mathrm{D}$ vector $\mathbf{r}=(x, y)$ represents a point in the planes of the membranes. We work in the low-Reynolds number regime of the membrane hydrodynamics so that the inertial effects can be neglected. This allows us to use the 2D Stokes equations

$$
\eta \nabla^{2} \mathbf{v}^{(i)}-\nabla p^{(i)}+\mathbf{f}_{\mathrm{s}}^{(i)}+\mathbf{F}^{(i)}=0,
$$

along with the incompressibility condition,

$$
\nabla \cdot \mathbf{v}^{(i)}=0 .
$$

Here $\nabla$ is a $2 \mathrm{D}$ differential operator, $\eta$ is the $2 \mathrm{D}$ membrane viscosity (same for both the membranes), $p^{(i)}(\mathbf{r})$ the 2D in-plane pressure, $\mathbf{f}_{\mathbf{s}}^{(i)}(\mathbf{r})$ the force exerted on the membrane by the surrounding fluid, and $\mathbf{F}^{(i)}(\mathbf{r})$ is any other force acting on the membrane. 
The solvent regions are denoted by the index $j=\mathrm{i}$, ii, iii. The velocities and pressures in these regions are written as $\mathbf{v}^{(j)}(\mathbf{r}, z)$ and $p^{(j)}(\mathbf{r}, z)$, respectively. We assume that the solvent in the three regions have the same $3 \mathrm{D}$ viscosity denoted by $\eta_{\mathbf{s}}$. The solvent inertia is neglected and hence it also obeys the 3D Stokes equations

$$
\eta_{\mathrm{s}} \tilde{\nabla}^{2} \mathbf{v}^{(j)}-\tilde{\nabla} p^{(j)}=0
$$

where $\tilde{\nabla}$ represents a 3D differential operator. Similar to the fluid membrane, the solvent in all the regions are considered to be incompressible

$$
\tilde{\nabla} \cdot \mathbf{v}^{(j)}=0 .
$$

The presence of the surrounding solvent is important because it exerts force on the liquid membranes. The force on membrane 1 , indicated as $\mathbf{f}_{\mathrm{s}}^{(1)}$ in (1), is given by the projection of $\left(\boldsymbol{\sigma}^{(\mathrm{i})}-\boldsymbol{\sigma}^{(\mathrm{ii})}\right)_{z=h / 2} \cdot \hat{\mathbf{e}}_{z}$ onto the $x y$-plane of the membrane. Here $\hat{\mathbf{e}}_{z}$ is the unit vector along the $z$-axis, and $\boldsymbol{\sigma}^{(j)}$ are the stress tensors

$$
\boldsymbol{\sigma}^{(j)}=-p^{(j)} \mathbf{I}+\eta_{\mathrm{s}}\left[\tilde{\nabla} \mathbf{v}^{(j)}+\left(\tilde{\nabla} \mathbf{v}^{(j)}\right)^{\mathrm{T}}\right] .
$$

In the above, $\mathbf{I}$ is the identity tensor and the superscript " $T$ " indicates the transpose. Similarly, the force on membrane 2 , is given by the projection of $\left(\boldsymbol{\sigma}^{(\mathrm{ii})}-\boldsymbol{\sigma}^{(\mathrm{iii})}\right)_{z=-h / 2} \cdot \hat{\mathbf{e}}_{z}$ on the $x y$-plane. The general procedure is to first resolve (1) into components along $\mathbf{k}$ and perpendicular to it, where $\mathbf{k}$ is the $2 \mathrm{D}$ wave vector in Fourier space. We then solve the resulting differential equations for the velocities. Stick boundary conditions at the membrane-solvent interfaces are imposed. It is also assumed that the solvent velocities decay to zero at sufficiently large distances from the membranes. A similar calculation for a single membrane confined between two walls has been previously performed [14, 15].

Owing to the linearity of governing Stokes equations, the in-plane velocity in membrane 1 can be obtained in Fourier space as

$$
v_{\alpha}^{(1)}[\mathbf{k}]=G_{\alpha \beta}^{(11)}[\mathbf{k}] F_{\beta}^{(1)}[\mathbf{k}]+G_{\alpha \beta}^{(12)}[\mathbf{k}] F_{\beta}^{(2)}[\mathbf{k}] .
$$

Here $G_{\alpha \beta}^{(11)}$ and $G_{\alpha \beta}^{(12)}(\alpha, \beta=x, y)$ are the mobility tensors given by

$$
\begin{aligned}
G_{\alpha \beta}^{(11)}[\mathbf{k}] & =\frac{1}{\eta \nu^{2}} \frac{1+2 K+\operatorname{coth}(K H)}{K g(K, H)}\left(\delta_{\alpha \beta}-\frac{k_{\alpha} k_{\beta}}{k^{2}}\right), \\
G_{\alpha \beta}^{(12)}[\mathbf{k}] & =\frac{1}{\eta \nu^{2}} \frac{\operatorname{cosech}(K H)}{K g(K, H)}\left(\delta_{\alpha \beta}-\frac{k_{\alpha} k_{\beta}}{k^{2}}\right),
\end{aligned}
$$

with

$$
g(K, H)=1+2 K(1+K)+(1+2 K) \operatorname{coth}(K H)
$$

and $\nu \equiv 2 \eta_{\mathrm{s}} / \eta$. Notice that $\nu^{-1}$ represents the hydrodynamic screening length. We have also used the definitions $H=h \nu$ and $K=k / \nu$ with $k=|\mathbf{k}|$. By symmetry, a similar set of expressions can be written down for the membrane 2 also. The above mobility tensors represent the hydrodynamic coupling between the two membranes.

Consider a pair of particles embedded in the membrane undergoing Brownian motion separated by r. For sufficiently large enough times, the particle displacements 


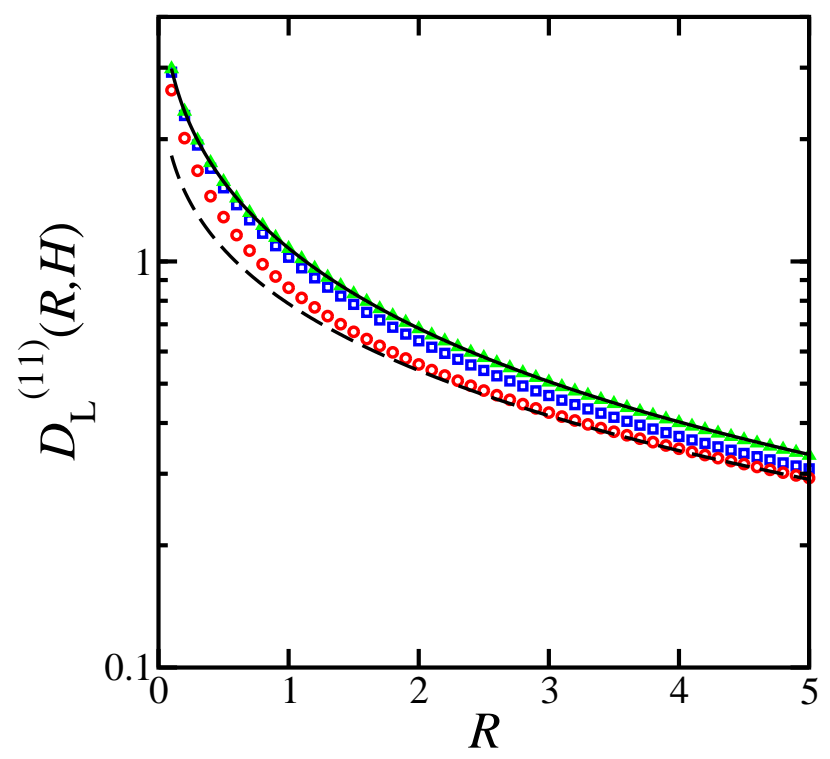

Figure 2. Dimensionless $D_{\mathrm{L}}^{(11)}(R, H)$ as a function of $R$ for various values of $H$. The red circles, blue squares and green triangles correspond to $H=0.1,1$ and 10, respectively (color online). The solid line corresponds to (11) representing the analytical expression for large $H$ limit. The dashed line corresponds to the small $H$ limit.

obey $\left\langle\Delta r_{\alpha} \Delta r_{\beta}^{\prime}\right\rangle=2 D_{\alpha \beta}(\mathbf{r}) t$ where $\Delta r_{\alpha}$ represents the displacement of the first particle and $\Delta r_{\beta}^{\prime}$ represents that of the second particle. In the above, the diffusion tensor for over-damped dynamics is given by the Einstein relation $D_{\alpha \beta}=k_{\mathrm{B}} T G_{\alpha \beta}$ where $k_{\mathrm{B}}$ is the Boltzmann constant and $T$ the temperature. We now apply these definitions to a double-membrane system. The line of centers connecting any two particles in the membranes after projection on to the $2 \mathrm{D}$ plane can be taken to be along the $x$-axis without loss of generality. Then we obtain the coupling longitudinal diffusion coefficients as $D_{\mathrm{L}}^{(11)}(\mathbf{r})=k_{\mathrm{B}} T G_{x x}^{(11)}\left(r \hat{e}_{x}\right)$ of two particles within the same membrane, and $D_{\mathrm{L}}^{(12)}(\mathbf{r})=$ $k_{\mathrm{B}} T G_{x x}^{(12)}\left(r \hat{e}_{x}\right)$ of two particles in different membranes. The coupling transverse diffusion coefficients are $D_{\mathrm{T}}^{(11)}(\mathbf{r})=k_{\mathrm{B}} T G_{y y}^{(11)}\left(r \hat{e}_{x}\right)$ and $D_{\mathrm{T}}^{(12)}(\mathbf{r})=k_{\mathrm{B}} T G_{y y}^{(12)}\left(r \hat{e}_{x}\right)$. The longitudinal coupling diffusion coefficient is associated with Brownian motion along the line of centers, while the transverse one is associated with motion perpendicular to the line of centers [11, 12, 16]. In the following, we discuss the above four diffusion coefficients sequentially.

The real-space expressions for the mobility tensors can be obtained by inverse Fourier transform. Using the notation $R=r \nu$, we obtain the longitudinal coupling diffusion coefficient within the same membrane as

$$
D_{\mathrm{L}}^{(11)}(R, H)=2 \int_{0}^{\infty} \mathrm{d} K \frac{1+2 K+\operatorname{coth}(K H)}{g(K, H)} \frac{J_{1}(K R)}{K R},
$$

where $J_{n}(t)$ are the Bessel function of the first kind of order $n$. Hereafter, all the diffusion coefficients are scaled by a factor $k_{\mathrm{B}} T /(4 \pi \eta)$ in order to make them dimensionless. In figure 2, we plot $D_{\mathrm{L}}^{(11)}(R, H)$ as a function of $R$ for various $H$ following numerical 


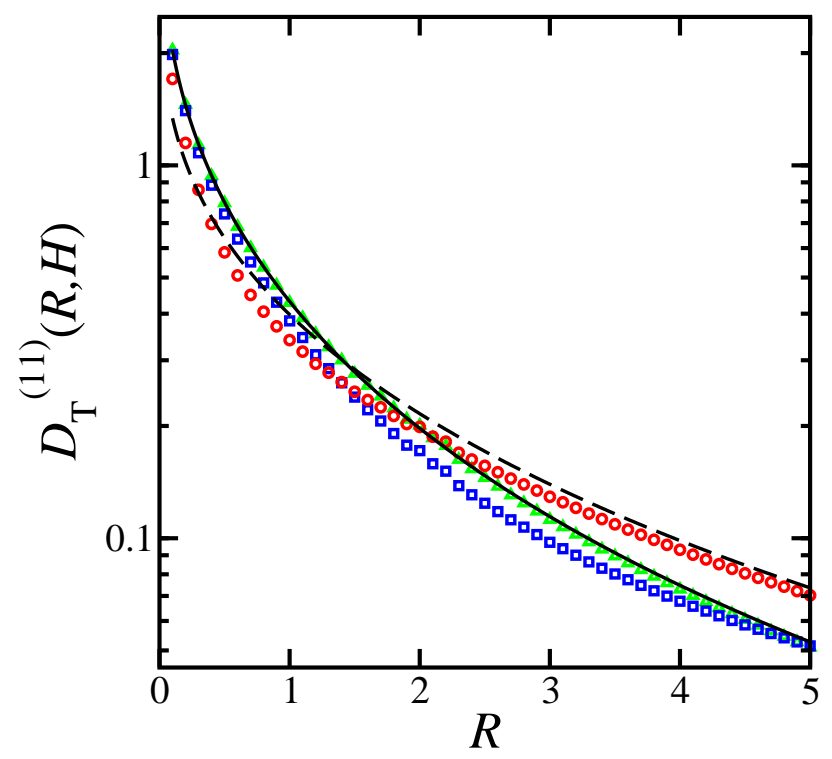

Figure 3. Dimensionless $D_{\mathrm{T}}^{(11)}(R, H)$ as a function of $R$ for various values of $H$. The red circles, blue squares and green triangles correspond to $H=0.1,1$ and 10, respectively (color online). The solid line corresponds to (13) representing the analytical expression for large $H$ limit. The dashed line corresponds to the small $H$ limit.

integration. It is a monotonically decreasing function of $R$. The red circles $(H=0.1)$, blue squares $(H=1)$ and green triangles $(H=10)$ represent the intermediate membrane separations respectively. These symbols represent the same $H$ values in the rest of the figures in this Communication.

For $H \gg 1$, we can approximate $\operatorname{coth}(K H) \approx 1$ so that the $H$-dependence drops out. In this limit, the integral can be analytically performed to yield

$$
D_{\mathrm{L}}^{(11)}(R)=-\frac{2}{R^{2}}+\frac{\pi}{R}\left[\mathbf{H}_{1}(R)-Y_{1}(R)\right],
$$

where $\mathbf{H}_{n}(t)$ are the Struve functions and $Y_{n}(t)$ are the Neumann functions. Since the membranes are effectively isolated from each other for large $H$, (11) coincides with the analytical expression obtained for a single membrane geometry [11]. For $R \ll 1$, (11) has a logarithmic behavior, i.e., $D_{\mathrm{L}}^{(11)}(R \ll 1) \approx \ln (2 / R)-\gamma+0.5$ where $\gamma=0.5772 \ldots$ is the Euler's constant. In this regime, the outer solvent surrounding the membrane is unimportant and the membrane behaves effectively as a pure $2 \mathrm{D}$ fluid. In the opposite $R \gg 1$ limit, (11) decays algebraically $D_{\mathrm{L}}^{(11)}(R \gg 1) \approx 2 / R$. On recasting in dimensional form, we see that this limiting expression is independent of the membrane viscosity $\eta$, and dependent only on the solvent viscosity $\eta_{\mathrm{s}}$. When $H \ll 1$, on the other hand, (10) becomes $D_{\mathrm{L}}^{(11)}(R / 2) / 2$. The vanishing thickness of the solvent region ii results in a rescaling of $\nu^{-1}$ by a factor of two, and hence the resultant expression. The solid and dashed lines in figure 2 represent the above limiting cases of large and small $H$ limits, respectively. It is thus observed that the presence of the second membrane also has a finite contribution to the coupling longitudinal diffusion coefficient within the same membrane. 


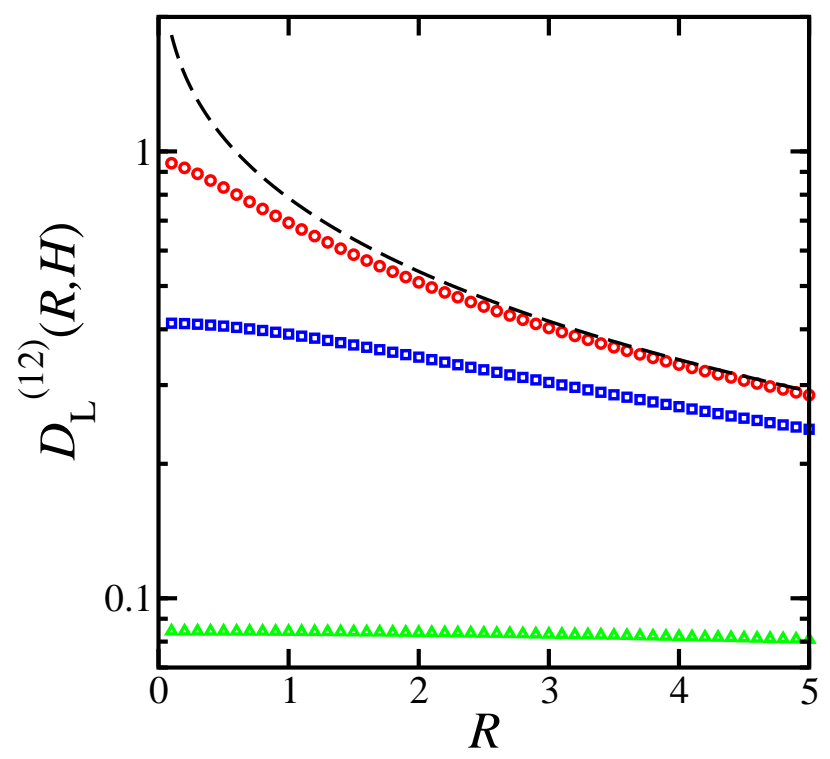

Figure 4. Dimensionless $D_{\mathrm{L}}^{(12)}(R, H)$ as a function of $R$ for various values of $H$. The red circles, blue squares and green triangles correspond to $H=0.1,1$ and 10 , respectively (color online). The dashed line corresponds to the small $H$ limit.

Following the same argument, the transverse coupling diffusion coefficient between two particles within the same membrane is calculated according to

$$
\begin{array}{rl}
D_{\mathrm{T}}^{(11)}(R, H)=2 \int_{0}^{\infty} \mathrm{d} & K \frac{1+2 K+\operatorname{coth}(K H)}{g(K, H)} \\
\times & {\left[J_{0}(K R)-\frac{J_{1}(K R)}{K R}\right] .}
\end{array}
$$

In figure 3, the variation of $D_{\mathrm{T}}^{(11)}(R, H)$ as a function of $R$ for various $H$ is plotted using numerical integration. Similar to the longitudinal case, it is also a monotonically decreasing function of $R$. For $H \gg 1$, (12) can be analytically treated to have the form

$$
\begin{aligned}
D_{\mathrm{T}}^{(11)}(R)= & \frac{2}{R^{2}}-\frac{\pi}{R}\left[\mathbf{H}_{1}(R)-Y_{1}(R)\right] \\
& +\pi\left[\mathbf{H}_{0}(R)-Y_{0}(R)\right],
\end{aligned}
$$

which also coincides with the expression for the transverse coupling diffusion coefficient in a single membrane [11. In this case, the asymptotic behaviors for small and large $R$ are $D_{\mathrm{T}}^{(11)}(R \ll 1) \approx \ln (2 / R)-\gamma-0.5$ and $D_{\mathrm{T}}^{(11)}(R \gg 1) \approx 2 / R^{2}$, respectively. In the opposite limit of $H \ll 1$, (12) becomes $D_{\mathrm{T}}^{(11)}(R / 2) / 2$. As before, the solid and dashed lines in figure 3 show the limiting cases of large and small $H$, respectively.

Now we proceed to calculate the coupling diffusion coefficients of two particles in different membranes. In this case, the longitudinal coupling diffusion coefficient expressed in dimensionless units is

$$
D_{\mathrm{L}}^{(12)}(R, H)=2 \int_{0}^{\infty} \mathrm{d} K \frac{\operatorname{cosech}(K H)}{g(K, H)} \frac{J_{1}(K R)}{K R} .
$$

The functional dependence of $D_{\mathrm{L}}^{(12)}(R, H)$ on $R$ for various $H$ is shown in figure 4 . 


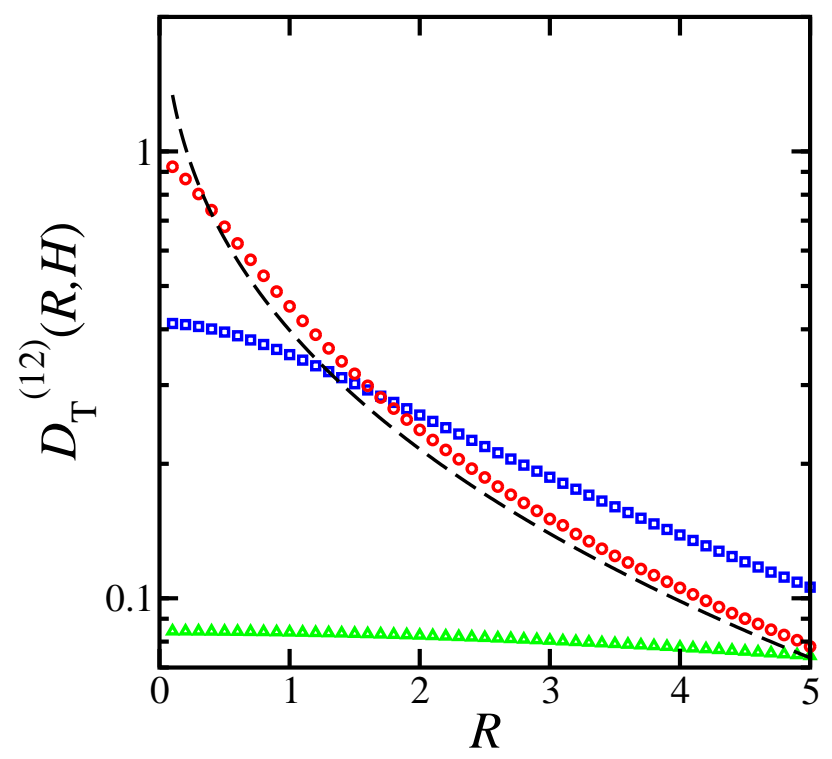

Figure 5. Dimensionless $D_{\mathrm{T}}^{(12)}(R, H)$ as a function of $R$ for various values of $H$. The red circles, blue squares and green triangles correspond to $H=0.1,1$ and 10, respectively (color online). The dashed line corresponds to the small $H$ limit.

Since $\operatorname{cosech}(K H) \approx 0$ for $H \gg 1$, the above integral vanishes for large inter-membrane distances as seen by the green triangles $(H=10)$ in figure 4. This is a reasonable result as the membranes are effectively independent of each other. In the limit of $H \ll 1$, (14) results in $D_{\mathrm{L}}^{(11)}(R / 2) / 2$ which is plotted by the dashed line in figure 4. As mentioned earlier, this function has an initial logarithmic behavior followed by an asymptotic $1 / R$ decay.

The transverse coupling diffusion coefficient between two particles in different membranes is given by

$$
\begin{aligned}
D_{\mathrm{T}}^{(12)}(R, H)=2 \int_{0}^{\infty} & \mathrm{d} K \frac{\operatorname{cosech}(K H)}{g(K, H)} \\
& \times\left[J_{0}(K R)-\frac{J_{1}(K R)}{K R}\right] .
\end{aligned}
$$

In figure 5, the variation of $D_{\mathrm{T}}^{(12)}(R, H)$ as a function of $R$ for various $H$ is plotted. The above integral also vanishes when $H \gg 1$ as expected for the decoupled membranes. In the opposite limit of $H \ll 1$, (15) results in $D_{\mathrm{T}}^{(11)}(R / 2) / 2$ as plotted by the dashed line in figure 5 .

From figures 4 and 5, it can be observed that for large $H$, the coupling diffusion coefficients vanish. This is due to the exponential decay of the $\operatorname{cosech}(K H)$ term. Up to $H=1$, the proximity to the second membrane leads to additional contributions to the coupling diffusion coefficients. This is the main result of this work. Using typical values for the solvent (water) and membrane (lipid bilayer) viscosities, the hydrodynamic screening length $\nu^{-1}$ can be estimated to be of the order of $\mu \mathrm{m}$. This implies that an adjacent membrane within this distance $(H<1)$ can have a strong bearing on the 
diffusion dynamics such as for typical stacked supported membrane experiments [9]. Qualitatively, the presence of the second membrane can enhance the effective coupling diffusion.

Even though the model presented in this Communication captures the essential physics, it looks somewhat simplistic in several respects. We have neglected the finite size effect of the membrane inclusions which are known to modify the membrane response at small inter-particle distances [11, 12]. At distances much larger than the inclusion size, however, these effects become unimportant. Curvature effects of the membrane is significant when the radius of curvature is of the order of the hydrodynamic screening length $\nu^{-1}$ [17, 18]. The extension of our work to membranes in spherical configuration is possible. The out-of-plane fluctuations of the membrane lead to a reduction in the diffusion coefficient of proteins in the single membranes [19, 20]. However, it is known that the presence of a substrate or the second membrane would suppress the out-of-plane membrane fluctuations [21, 22]. Overall, we expect that fluctuations and the presence of a substrate will not qualitatively affect our results.

To summarize, we have calculated the longitudinal as well as transverse coupling diffusion coefficients of particles undergoing Brownian motion in a stacked double membrane geometry. We obtained the additional contribution to the coupling diffusion coming from the proximity to adjacent membranes. As in biological systems such as tissues, the cells are rarely isolated, our results also imply that the proximity to neighboring cells can affect the diffusion of objects in cell membranes.

\section{Acknowledgments}

This work was supported by KAKENHI (Grant-in-Aid for Scientific Research) on

Priority Area "Soft Matter Physics" and Grant No. 21540420 from the Ministry of Education, Culture, Sports, Science and Technology of Japan.

\section{References}

[1] B. Alberts, A. Johnson, P. Walter, J. Lewis, and M. Raff. Molecular Biology of the Cell. Garland Science, New York, 2008.

[2] P. G. Saffman and M. Delbrück. Brownian motion in biological membranes. Proc. Natl. Acad. Sci. USA, 72:3111-3113, 1975.

[3] E. Evans and E. Sackmann. Translational and rotational drag coefficients for a disk moving in a liquid membrane associated with a rigid substrate. J. Fluid Mech., 194:553-561, 1988.

[4] D. K. Lubensky and R. E. Goldstein. Hydrodynamics of monolayer domains at the air-water interface. Phys. Fluids, 8:843-854, 1996.

[5] S. Ramachandran, S. Komura, M. Imai, and K. Seki. Drag coefficient of a liquid domain in a two-dimensional membrane. Eur. Phys. J. E, 31:303-310, 2010.

[6] A. J. Levine, T. B. Liverpool, and F. C. MacKintosh. Dynamics of rigid and flexible extended bodies in viscous films and membranes. Phys. Rev. Lett., 93:038102, 2004.

[7] A. J. Levine, T. B. Liverpool, and F. C. MacKintosh. Mobility of extended bodies in viscous films and membranes. Phys. Rev. E, 69:021503, 2004. 
[8] Th. M. Fischer. The drag on needles moving in a Langmuir monolayer. J. Fluid Mech., 498:123137, 2004.

[9] Y. Kaizuka and J. T. Groves. Structure and dynamics of supported intermembrane junctions. Biophys. J., 86:905-912, 2004.

[10] J. T. Groves. Bending mechanics and molecular organization in biological membranes. Annu. Rev. Phys. Chem., 58:697-717, 2007.

[11] N. Oppenheimer and H. Diamant. Correlated diffusion of membrane proteins and their effect on membrane viscosity. Biophys. J., 96:3041-3049, 2009.

[12] N. Oppenheimer and H. Diamant. Correlated dynamics of inclusions in a supported membrane. Phys. Rev. E, 82:041912, 2010.

[13] P. G. de Gennes and F. Brochard. Microemulsions and the flexibility of oil/water interfaces. J. Phys. Chem., 86:2294-2304, 1982.

[14] S. Ramachandran, S. Komura, K. Seki, and M. Imai. Hydrodynamic effects on concentration fluctuations in multicomponent membranes. Accepted in Soft Matter, DOI: 10.1039/c0sm00783h, 2011.

[15] S. Ramachandran, S. Komura, K. Seki, and G. Gompper. Dynamics of a polymer chain confined in a membrane. Preprint, 2010.

[16] S. Ramachandran, S. Komura, and G. Gompper. Effects of an embedding bulk fluid on phase separation dynamics in a thin liquid film. Europhys. Lett., 89:56001, 2010.

[17] M. L. Henle, R. McGotry, A. B. Schofield, A. D. Dinsmore, and A. J. Levine. The effect of curvature and topology on membrane hydrodynamics. Europhys. Lett., 84:48001, 2008.

[18] M. L. Henle and A. J. Levine. Hydrodynamics in curved membranes: The effect of geometry on particulate mobility. Phys. Rev. E, 81:011905, 2010.

[19] A. Naji, P. J. Atzberger, and F. L. H. Brown. Hybrid elastic and discrete-particle approach to biomembrane dynamics with application to the mobility of curved integral membrane proteins. Phys. Rev. Lett., 102:138102, 2009.

[20] E. Reister-Gottfried, S. M. Leitenberger, and U. Seifert. Diffusing proteins on a fluctuating membrane: Analytical theory and simulations. Phys. Rev. E, 81(3):031903, 2010.

[21] S. Sankararaman, G. I. Menon, and P. B. S. Kumar. Two-component fluid membranes near repulsive walls: Linearized hydrodynamics of equilibrium and nonequilibrium states. Phys. Rev. E, 66:031914, 2002.

[22] N. Gov, A. G. Zilman, and S. Safran. Hydrodynamics of confined geometries. Phys. Rev. E, 70:011104, 2004. 